\title{
O papel dos inibidores da fosfodiesterase 5 no tratamento dos sintomas do trato urinário inferior: revisão baseada na evidência
}

Hugo Rocha,* Ângela Neves,* Sofia Fernandes*

\section{RESUMO}

Objetivo: Determinar a evidência existente acerca da eficácia e segurança do uso dos inibidores da fosfodiesterase 5 (IPDE5) em monoterapia nos sintomas do trato urinário inferior (LUTS) secundários a hipertrofia benigna prostática, com ou sem disfunção eréctil associada.

Fontes de dados: MEDLINE e sítios de medicina baseada na evidência.

Métodos de revisão: Pesquisa de normas de orientação clínica (NOC), meta-analises (MA), revisões sistemáticas (RS) e ensaios clínicos aleatorizados (ECA), utilizando os termos MeSH: Phosphodiesterase 5 inhibitors, Lower urinary tract symptoms, Prostatic hyperplasia. Para avaliar a qualidade dos estudos e a força de recomendação foi utilizada a escala Strength of Recommendation Taxonomy (SORT), da American Family Physician.

Resultados: Foram identificados 172 artigos, dos quais foram selecionados oito. Uma NOC, cinco MAs, uma RS e um ECA cumpriram os critérios de inclusão. A utilização de IPDE5 demonstrou melhoria significativa da pontuação no Internacional Prostatic Symptom Score (IPSS) e no Índice Internacional de Função Eréctil (IIFE) e associou-se a uma maior incidência global de efeitos adversos (EA) leves a moderados, sem diferenças nos EA graves, com perfil de segurança favorável.

Conclusões: Os resultados obtidos apresentam evidência robusta acerca da eficácia e segurança do uso dos IPDE5 nos LUTS, com ou sem disfunção eréctil associada (SORT A). Sugere-se a realização de mais estudos para a avaliar a eficácia e segurança a longo prazo, o seu papel na progressão da doença e a relação custo-efetividade da sua utilização nos LUTS.

Palavras-chave: Sintomas do trato urinário inferior; LUTS; Inibidores da fosfodiesterase 5; Hipertrofia benigna da próstata.

\section{INTRODUÇÃO}

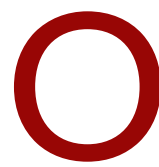

s sintomas do trato urinário inferior, comumente designados de LUTS (acrónimo para a expressão inglesa Lower urinary tract symptoms), têm um considerável impacto na qualidade de vida, representando um dos principais motivos de procura de cuidados médicos pelo homem adulto. A prevalência dos LUTS varia entre 10 a $25 \%,{ }^{1}$ encontrando-se descritas prevalências de $40 \%$ aos 50 anos de idade e de $50 \%$ aos 80 anos. $^{2} \mathrm{O}$ envelhecimento da população com aumento da esperança média de vida faz prever um aumento da procura de cuidados médicos por queixas associadas aos LUTS.

Dividem-se essencialmente em dois grandes grupos:

* Médicos internos de formação específica em Medicina Geral e Familiar ULS Matosinhos - USF Lagoa sintomas de armazenamento (urgência urinária, polaquiúria e noctúria) e sintomas obstrutivos (hesitação, jato urinário fino, fraco, gotejante ou interrompido e sensação de micção incompleta). ${ }^{2}$

Os LUTS podem ser quantificados segundo um questionário validado: o International Prostatic Symptom Score (IPSS). Este é composto por sete perguntas que avaliam a ocorrência de sintomas no mês anterior à data da sua realização e uma pergunta sobre qualidade de vida (IPSS-QoL) - quadro I. As perguntas número dois, quatro e sete avaliam sintomas de armazenamento, pelo que podem ser consideradas como «IPSS subscore de armazenamento». As questões número um, três, cinco e seis avaliam os sintomas obstrutivos, pelo que se consideram como «IPSS subscore miccional». ${ }^{3}$ Uma pontuação no IPSS total 


\begin{tabular}{|c|c|c|c|c|c|c|c|c|}
\hline \multicolumn{2}{|l|}{ Sintomas/Pontuação } & $\begin{array}{l}\text { Nenhuma } \\
\text { vez }\end{array}$ & $\begin{array}{c}\text { Menos de } 1 \\
\text { em cada } 5 \\
\text { vezes }\end{array}$ & $\begin{array}{c}\text { Menos de } \\
\text { metade } \\
\text { das vezes }\end{array}$ & $\begin{array}{l}\text { Metade } \\
\text { das vezes }\end{array}$ & \multicolumn{2}{|c|}{$\begin{array}{c}\text { Mais de } \\
\text { metade } \\
\text { das vezes }\end{array}$} & $\begin{array}{l}\text { Quase } \\
\text { sempre }\end{array}$ \\
\hline \multicolumn{2}{|l|}{1 - Sensação de micção incompleta? } & 0 & 1 & 2 & 3 & & 4 & 5 \\
\hline \multicolumn{2}{|c|}{$\begin{array}{l}2 \text { - Urina dentro de duas horas depois da última } \\
\text { micção? }\end{array}$} & 0 & 1 & 2 & 3 & & 4 & 5 \\
\hline \multicolumn{2}{|c|}{ 3- Começa e termina o jato urinário várias vezes? } & 0 & 1 & 2 & 3 & & 4 & 5 \\
\hline \multicolumn{2}{|l|}{4 - Dificuldade em conter uma micção? } & 0 & 1 & 2 & 3 & & 4 & 5 \\
\hline \multicolumn{2}{|l|}{5 - Jato urinário mais fraco? } & 0 & 1 & 2 & 3 & & 4 & 5 \\
\hline \multirow{2}{*}{\multicolumn{2}{|c|}{6 - Faz força para começar a urinar? }} & 0 & 1 & 2 & 3 & & 4 & 5 \\
\hline & & $\begin{array}{l}\text { Nenhuma } \\
\text { vez }\end{array}$ & $1 \mathrm{vez}$ & 2 vezes & 3 vezes & & ezes & $\begin{array}{l}5 \text { vezes } \\
\text { ou mais }\end{array}$ \\
\hline \multicolumn{2}{|c|}{$\begin{array}{l}7 \text { - Quantas vezes tem de se levantar durante a } \\
\text { noite para urinar? }\end{array}$} & 0 & 1 & 2 & 3 & & 4 & 5 \\
\hline Qualidade de Vida & Ótimo & Muito bem & Satisfeito & Mais ou menos & \multicolumn{2}{|c|}{ Insatisfeito } & Mal & Péssimo \\
\hline $\begin{array}{l}\text { Se tivesse que passar o resto da sua vida } \\
\text { com estes sintomas, como se sentiria? }\end{array}$ & 0 & 1 & 2 & 3 & \multicolumn{2}{|l|}{4} & 5 & 6 \\
\hline
\end{tabular}

entre zero e sete valores corresponde a sintomatologia leve, entre oito e dezanove a sintomatologia moderada e, entre vinte e trinta e cinco, a sintomatologia severa. ${ }^{2}$ Os doentes com uma pontuação no IPSS superior a oito têm indicação para tratamento farmacológico. ${ }^{2}$

Existe uma relação comprovada entre a disfunção eréctil (DE) e os LUTS. ${ }^{4-8}$ Cerca de $70 \%$ dos homens com LUTS têm também DE. ${ }^{4}$ Em termos fisiopatológicos, embora não sejam completamente conhecidos os mecanismos que relacionam ambas as patologias, partilham alterações em vias comuns. . $^{5-6}$

A disfunção eréctil pode ser avaliada mediante aplicação de um questionário validado: o International Index of Erectile function (IIFE) - quadro II. É composto por 15 perguntas e avalia todos os domínios da função sexual masculina: função eréctil, função orgástica, desejo sexual, ejaculação, coito e satisfação geral. O IIFE classifica a gravidade da DE em cinco categorias: ausência de DE (26 a 30), leve (22 a 25), leve a moderada (17 a 21 ), moderada (11 a 16) e grave (6 a 10). ${ }^{9}$

Os principais fármacos utilizados para tratar os LUTS secundários a HBP são os alfa-bloqueantes $(\mathrm{AB})$, os inibidores da 5 alfa reductase (i5AR) e os antagonistas dos recetores muscarínicos (ARM). Apesar dos AB serem considerados o tratamento de $1 .{ }^{\mathrm{a}}$ linha nos LUTS secundários a $\mathrm{HBP}^{2}$ estes estão associados a efeitos adversos (EA) como: náuseas, hipotensão ortostática e disfunção sexual (disfunção eréctil e ejaculação anormal). Assim, são necessários tratamentos alternativos para os homens que não to- leram ou não respondem a estes fármacos, bem como para todos aqueles em que os potenciais efeitos colaterais destes possam constituir uma importante preocupação.

A primeira evidência do papel dos inibidores da fosfodiesterase 5 (IPDE5) nos LUTS surgiu de estudos feitos em homens com disfunção eréctil, onde ocorreu uma melhoria secundária dos sintomas do trato urinário. ${ }^{10}$ Esta ação farmacológica resulta da distribuição da fosfodiesterase 5 na bexiga, uretra e próstata (por ordem decrescente de concentração orgânica desta enzima), pelo que podem aumentar o cGMP intracelular, relaxando o músculo liso do detrusor, próstata e uretra. Tambémé sugerido um papel destes fármacos na melhoria da circulação sanguínea nos vasos pélvicos por relaxamento do músculo liso e modulação do sistema nervoso autónomo por via dos nervos aferentes da bexiga e próstata. ${ }^{6}$

Diversas investigações têm sugerido a utilidade dos IPDE5 nos LUTS, em pacientes com ou sem DE, ${ }^{5,11-13}$ pelo que esta classe farmacológica tem emergido recentemente como nova opção terapêutica para o tratamento simultâneo destas duas patologias tão frequentemente associadas. Desde 2011 encontra-se aprovado o uso do tadalafil no tratamento dos LUTS com ou sem disfunção eréctil na Europa (European Medicines Agency - EMA/647346/2012).

No entanto, embora o tadalafil tenha sido aprovado pela EMA, várias questões têm limitado a sua aplicabilidade neste âmbito: eficácia indeterminada, resultados inexplorados e evidência ainda não estabelecida, pelo que a sua 
QUADRO II. Questionário IIFE - Índice Internacional de Função Eréctil

Nas últimas 4 semanas...

\begin{tabular}{|c|c|c|c|c|c|c|}
\hline $\begin{array}{l}\text { 1) Com que frequência conseguiu } \\
\text { uma ereção durante a atividade } \\
\text { sexual? }\end{array}$ & $\begin{array}{l}\text { Não tive } \\
\text { relações } \\
\text { sexuais "0" }\end{array}$ & $\begin{array}{l}\text { Quase nunca } \\
\text { ou nunca } \\
\text { "1" }\end{array}$ & $\begin{array}{l}\text { Menos de } \\
\text { metade das } \\
\text { vezes "2" }\end{array}$ & $\begin{array}{l}\text { Metade das } \\
\text { vezes " } 3 \text { " }\end{array}$ & $\begin{array}{l}\text { Mais de } \\
\text { metade das } \\
\text { vezes " } 4 \text { " }\end{array}$ & $\begin{array}{l}\text { Quase } \\
\text { sempre ou } \\
\text { sempre " } 5 \text { " }\end{array}$ \\
\hline $\begin{array}{l}\text { 2) Quando teve ereções com } \\
\text { estimulação sexual, com que } \\
\text { frequência as ereções foram } \\
\text { suficientes para permitir } \\
\text { penetração? }\end{array}$ & $\begin{array}{l}\text { Não tive } \\
\text { relações } \\
\text { sexuais "0" }\end{array}$ & $\begin{array}{l}\text { Quase nunca } \\
\text { ou nunca "1" }\end{array}$ & $\begin{array}{l}\text { Menos de } \\
\text { metade das } \\
\text { vezes "2" }\end{array}$ & $\begin{array}{l}\text { Metade das } \\
\text { vezes " } 3 \text { " }\end{array}$ & $\begin{array}{l}\text { Mais de } \\
\text { metade das } \\
\text { vezes " } 4 \text { " }\end{array}$ & $\begin{array}{l}\text { Quase } \\
\text { sempre ou } \\
\text { sempre " } 5 \text { " }\end{array}$ \\
\hline $\begin{array}{l}\text { 3) Quando tentou ter relações } \\
\text { sexuais, com que frequência } \\
\text { conseguiu penetrar a(o) } \\
\text { parceira(o)? }\end{array}$ & $\begin{array}{l}\text { Não tive } \\
\text { relações } \\
\text { sexuais "0" }\end{array}$ & $\begin{array}{l}\text { Quase nunca } \\
\text { ou nunca "1" }\end{array}$ & $\begin{array}{l}\text { Menos de } \\
\text { metade das } \\
\text { vezes " } 2 \text { " }\end{array}$ & $\begin{array}{l}\text { Metade das } \\
\text { vezes " } 3 \text { " }\end{array}$ & $\begin{array}{l}\text { Mais de } \\
\text { metade das } \\
\text { vezes " } 4 \text { " }\end{array}$ & $\begin{array}{l}\text { Quase } \\
\text { sempre ou } \\
\text { sempre " } 5 \text { " }\end{array}$ \\
\hline $\begin{array}{l}\text { 4) Durante a relação sexual, com } \\
\text { que frequência conseguiu manter } \\
\text { a ereção depois da penetração? }\end{array}$ & $\begin{array}{l}\text { Não tive } \\
\text { relações } \\
\text { sexuais "0" }\end{array}$ & $\begin{array}{l}\text { Quase nunca } \\
\text { ou nunca "1" }\end{array}$ & $\begin{array}{l}\text { Menos de } \\
\text { metade das } \\
\text { vezes "2" }\end{array}$ & $\begin{array}{l}\text { Metade das } \\
\text { vezes " } 3 \text { " }\end{array}$ & $\begin{array}{l}\text { Mais de } \\
\text { metade das } \\
\text { vezes " } 4 \text { " }\end{array}$ & $\begin{array}{l}\text { Quase } \\
\text { sempre ou } \\
\text { sempre " } 5 \text { " }\end{array}$ \\
\hline $\begin{array}{l}\text { 5) Durante a relação sexual, quão } \\
\text { difícil foi manter a ereção para } \\
\text { até terminar a relação sexual? }\end{array}$ & $\begin{array}{l}\text { Não tive } \\
\text { relações } \\
\text { sexuais "0" }\end{array}$ & $\begin{array}{l}\text { Extrema- } \\
\text { mente difícil } \\
\text { "1" }\end{array}$ & $\begin{array}{l}\text { Muito difícil } \\
\text { "2" }\end{array}$ & Difícil "3" & $\begin{array}{l}\text { Ligeiramente } \\
\text { difícil "4" }\end{array}$ & $\begin{array}{l}\text { Sem } \\
\text { dificuldade } \\
\text { "5" }\end{array}$ \\
\hline $\begin{array}{l}\text { 6) Quantas vezes tentou ter } \\
\text { relações sexuais? }\end{array}$ & $\begin{array}{l}\text { Nenhuma } \\
\text { vez "0" }\end{array}$ & $\begin{array}{l}\text { Uma ou duas } \\
\text { tentativas } \\
\text { "1" }\end{array}$ & $\begin{array}{l}\text { Três ou } \\
\text { quatro } \\
\text { tentativas "2" }\end{array}$ & \begin{tabular}{|l|} 
Cinco ou \\
seis \\
tentativas "3"
\end{tabular} & $\begin{array}{l}\text { Sete a } 10 \\
\text { tentativas } \\
\text { " } 4 \text { " }\end{array}$ & $\begin{array}{l}11 \text { ou mais } \\
\text { tentativas } \\
\text { " } 5 \text { " }\end{array}$ \\
\hline $\begin{array}{l}\text { 7) Quando tentou ter relações } \\
\text { sexuais, com que frequência } \\
\text { foram satisfatórias? }\end{array}$ & $\begin{array}{l}\text { Não tive } \\
\text { relações } \\
\text { sexuais "0" }\end{array}$ & $\begin{array}{l}\text { Quase nunca } \\
\text { ou nunca "1" }\end{array}$ & $\begin{array}{l}\text { Menos de } \\
\text { metade das } \\
\text { vezes " } 2 \text { " }\end{array}$ & $\begin{array}{l}\text { Metade das } \\
\text { vezes " } 3 \text { " }\end{array}$ & $\begin{array}{l}\text { Mais de } \\
\text { metade das } \\
\text { vezes " } 4 \text { " }\end{array}$ & $\begin{array}{l}\text { Quase } \\
\text { sempre ou } \\
\text { sempre " } 5 \text { " }\end{array}$ \\
\hline $\begin{array}{l}\text { 8) Quão satisfeito ficou com as } \\
\text { relações sexuais? }\end{array}$ & $\begin{array}{l}\text { Não tive } \\
\text { relações } \\
\text { sexuais "0" }\end{array}$ & $\begin{array}{l}\text { Nenhuma } \\
\text { satisfação } \\
\text { "1" }\end{array}$ & $\begin{array}{l}\text { Pouca } \\
\text { satisfação } \\
\text { "2" }\end{array}$ & $\begin{array}{l}\text { Alguma } \\
\text { satisfação } \\
\text { "3" }\end{array}$ & $\begin{array}{l}\text { Moderada } \\
\text { satisfação } \\
\text { "4" }\end{array}$ & $\begin{array}{l}\text { Muita } \\
\text { satisfação } \\
\text { "5" }\end{array}$ \\
\hline $\begin{array}{l}\text { 9) Quando teve estimulação } \\
\text { sexual ou relação sexual, com } \\
\text { que frequência ejaculou? }\end{array}$ & $\begin{array}{l}\text { Não tive } \\
\text { relações } \\
\text { sexuais "0" }\end{array}$ & $\begin{array}{l}\text { Quase nunca } \\
\text { ou nunca "1" }\end{array}$ & $\begin{array}{l}\text { Menos de } \\
\text { metade das } \\
\text { vezes " } 2 \text { " }\end{array}$ & $\begin{array}{l}\text { Metade das } \\
\text { vezes " } 3 \text { " }\end{array}$ & $\begin{array}{l}\text { Mais de } \\
\text { metade das } \\
\text { vezes " } 4 \text { " }\end{array}$ & $\begin{array}{l}\text { Quase } \\
\text { sempre ou } \\
\text { sempre " } 5 \text { " }\end{array}$ \\
\hline $\begin{array}{l}\text { 10) Quando teve estimulação } \\
\text { sexual ou relação sexual, com que } \\
\text { frequência teve a sensação de } \\
\text { orgasmo ou clímax? }\end{array}$ & & $\begin{array}{l}\text { Quase nunca } \\
\text { ou nunca "1" }\end{array}$ & $\begin{array}{l}\text { Menos de } \\
\text { metade das } \\
\text { vezes "2" }\end{array}$ & $\begin{array}{l}\text { Metade das" } \\
\text { vezes "3 }\end{array}$ & $\begin{array}{l}\text { Mais de } \\
\text { metade das } \\
\text { vezes " } 4 \text { " }\end{array}$ & $\begin{array}{l}\text { Quase } \\
\text { sempre ou } \\
\text { sempre " } 5 \text { " }\end{array}$ \\
\hline $\begin{array}{l}\text { 11) Com que frequência sentiu } \\
\text { desejo sexual? }\end{array}$ & & $\begin{array}{l}\text { Quase nunca } \\
\text { ou nunca "1" }\end{array}$ & $\begin{array}{l}\text { Menos de } \\
\text { metade das } \\
\text { vezes "2" }\end{array}$ & $\begin{array}{l}\text { Metade das } \\
\text { vezes " } 3 \text { " }\end{array}$ & $\begin{array}{l}\text { Mais de } \\
\text { metade das } \\
\text { vezes "4" }\end{array}$ & $\begin{array}{l}\text { Quase } \\
\text { sempre ou } \\
\text { sempre " } 5 \text { " }\end{array}$ \\
\hline $\begin{array}{l}\text { 12) Como classificaria o seu nível } \\
\text { de desejo sexual? }\end{array}$ & & $\begin{array}{l}\text { Muito baixo ou } \\
\text { nenhum "1" }\end{array}$ & Baixo "2" & $\begin{array}{l}\text { Moderado } \\
\text { "3" }\end{array}$ & Alto "4" & $\begin{array}{l}\text { Muito alto } \\
\text { "5" }\end{array}$ \\
\hline $\begin{array}{l}\text { 13) Quão satisfeito tem estado } \\
\text { com a sua vida sexual, } \\
\text { globalmente? }\end{array}$ & & $\begin{array}{l}\text { Muito } \\
\text { insatisfeito } \\
\text { "1" }\end{array}$ & $\begin{array}{l}\text { Moderada- } \\
\text { mente } \\
\text { insatisfeito "2" }\end{array}$ & \begin{tabular}{|l|} 
Igualmente \\
satisfeito e \\
insatisfeito"3"
\end{tabular} & $\begin{array}{l}\text { Moderada- } \\
\text { mente } \\
\text { satisfeito " } 4 \text { " }\end{array}$ & $\begin{array}{l}\text { Muito } \\
\text { satisfeito } \\
\text { "5" }\end{array}$ \\
\hline $\begin{array}{l}\text { 14) Quão satisfeito tem estado } \\
\text { com a relação sexual que tem } \\
\text { com a(o) sua(eu) parceira(o)? }\end{array}$ & & $\begin{array}{l}\text { Muito } \\
\text { insatisfeito } \\
\text { "1" }\end{array}$ & \begin{tabular}{l|} 
Moderada- \\
mente \\
insatisfeito "2"
\end{tabular} & \begin{tabular}{|l|} 
Igualmente \\
satisfeito e \\
insatisfeito "3"
\end{tabular} & $\begin{array}{l}\text { Moderada- } \\
\text { mente } \\
\text { satisfeito "4" }\end{array}$ & $\begin{array}{l}\text { Muito } \\
\text { satisfeito "5" }\end{array}$ \\
\hline $\begin{array}{l}\text { 15) Como classifica a sua } \\
\text { confiança em ter e manter uma } \\
\text { ereção? }\end{array}$ & & $\begin{array}{l}\text { Muito baixa } \\
\text { "1" }\end{array}$ & Baixa "2" & $\begin{array}{l}\text { Moderada } \\
\text { "3" }\end{array}$ & Alta "4" & $\begin{array}{l}\text { Muito alta } \\
\text { "5" }\end{array}$ \\
\hline
\end{tabular}


utilização prática generalizada no tratamento dos LUTS tem apresentado uma aplicabilidade diminuta nos diferentes países, nomeadamente em Portugal.

Tendo em conta estes pressupostos, esta revisão baseada na evidência tem como objetivo determinar a evidência existente acerca da eficácia e segurança do uso dos IPDE5 em monoterapia nos LUTS secundários a HBP, com ou sem disfunção eréctil associada.

\section{MÉTODOS}

Tendo como ponto de partida as bases de dados MEDLINE, National Guidelines Clearinghouse, Guidelines Finder, Canadian Medical Association Practice Guidelines, Cochrane, DARE, Bandoliere referências bibliográficas dos artigos selecionados, foi feita uma pesquisa de normas de orientação clínica (NOC), meta-análises (MA), revisões sistemáticas (RS) e ensaios clínicos aleatorizados (ECAs). Foram utilizados os termos MeSH - Phosphodiesterase 5 inhibitors, Lower urinary tract symptoms, Prostatic hyperplasia-, tendo sido pesquisados artigos publicados desde janeiro de 2011 até maio de 2015 em língua inglesa, francesa, espanhola e portuguesa.

Os critérios utilizados para a inclusão dos artigos nesta revisão foram definidos segundo o Modelo PICO: População - doentes adultos com LUTS secundários a HBP, com uma pontuação superior a oito no questionário IPSS; Intervenção - administração de IPDE5 em monoterapia; Comparação - placebo ou terapêutica com alfa-bloqueante; Resultados - outcomes primários: melhoria da sintomatologia e qualidade de vida, avaliados pelo IPSS total, IPSS subscore miccional e IPSS subscore de armazenamento; melhoria da função eréctil, avaliada pelo IIFE; outcomes secundários: perfil de EA; relação custo-eficácia; efeitos a longo prazo.

Para avaliar a qualidade dos estudos e a força de recomendação foi utilizada a escala de Strength of Recommendation Taxonomy (SORT), da American Family Physician. ${ }^{14}$

\section{RESULTADOS}

Foram identificados 172 artigos, dos quais foram selecionados oito. Uma NOC, cinco MA, uma RS e um ECA cumpriram os critérios de inclusão. O processo de seleção dos estudos está representado na figura 1. 5 alfa reductase.
138 Artigos excluídos por:

- Temas não relacionados com o objetivo do trabalho;

- Revisões clássicas;

- Artigos editoriais;

- Artigos de informação ao público.

4 Artigos excluídos:

- Duas RS que avaliaram a eficácia do tratamento combinado com IPDE5 + AB vs placebo e vs IPDE5 (Intervenção e Comparação não concordante com PICO definido);

- Um ECA que avaliou eficácia do tratamento com IPDE5 na retenção urinária aguda secundária a HBP;

- Um ECA que avaliou a eficácia do tratamento combinado com IPDE5 + I5AR (intervenção não concordante com PICO definido).

Figura 1. Organigrama da seleção dos artigos. fodiesterase $5 ; A B$, alfa bloqueante; $H B P$, hipertrofia benigna prostática; I5AR, inibidores da

\section{Normas de orientação clínica}

A NOC incluída é referente às guidelines para a abordagem dos LUTS, da Sociedade Europeia de Urologia, atualizada em 2015. ${ }^{15}$ Atribuem uma força de recomendação A para a utilização dos IPDE5 nos homens com LUTS moderados a severos (evidência baseada na Oxford Center for Evidence - revisões sistemáticas com ECAs homogéneos e ECAs com intervalo de confiança estreito, todos com resultados consistentes). Esta recomendação é independente da existência ou não de disfunção eréctil. As guidelines reforçam que, na Europa, apenas o tadalafil $5 \mathrm{mg} /$ dia se encontra licenciado. Apesar destas recomendações, a eficácia e tolerabilidade após um ano não podem ser concluídas mediante ausência de estudos a longo prazo, não havendo informação suficiente sobre efeitos na redução do tamanho da próstata ou na progressão da doença.

\section{Meta-análises}

IPDE5 versus placebo

Nos estudos que compararam a utilização de IPDE5 com placebo verificou-se uma melhoria no IPSS total $(-2,8$; $p<0,0001 ;-2,12 ; p<0,01 ;-2,3 ; p<0,0001 ;-2,2 ; p=0,0007$; - 


\section{QUADRO III. Meta-análises (MA)}

\begin{tabular}{|c|c|c|c|c|}
\hline Referência & $\begin{array}{c}\text { Estudos, } \\
\text { População }\end{array}$ & Intervenção & Resultados & $\begin{array}{c}\text { Nível de } \\
\text { evidência }\end{array}$ \\
\hline $\begin{array}{l}\text { Gacci e } \\
\text { colaboradores } \\
(2012)^{6}\end{array}$ & $\begin{array}{l}7 \text { ECAs } \\
n=3.214 \\
\text { Duplamente } \\
\text { cegos } \\
12 \text { semanas }\end{array}$ & $\begin{array}{l}\text { IPDE5 (sildenafil ou tadalafil } \\
\text { ou vardenafil) } \\
\text { vs } \\
\text { Placebo }\end{array}$ & $\begin{array}{l}\text { A utilização de IPDE5 de forma isolada demonstrou } \\
\text { uma melhoria significativa da pontuação no IPSS } \\
(-2,8 ; p<0,0001) \text { e no IIFE }(+5,5 ; p<0,0001)\end{array}$ & 1 \\
\hline $\begin{array}{l}\text { Wang e } \\
\text { colaboradores } \\
(2014)^{16}\end{array}$ & $\begin{array}{l}12 \text { ECAs } \\
n=5.443 \\
\text { Duplamente } \\
\text { cegos } \\
7 \text { ECAs } \\
n=1.446 \\
\text { Duplamente } \\
\text { cegos }\end{array}$ & $\begin{array}{l}\text { 1.IPDE5 (sildenafil ou } \\
\text { tadalafil ou vardenafil) } \\
\text { vs } \\
\text { Placebo } \\
\text { 2.IPDE5s (sildenafil ou } \\
\text { tadalafil ou vardenafil) } \\
\text { vs } \\
\text { Alfa bloqueante (solifenacina, } \\
\text { tansulosina, alfuzosina, } \\
\text { prazosina, terazosina, } \\
\text { doxazosina) }\end{array}$ & $\begin{array}{l}\text { 1. IPDE5 isoladamente mostrou melhoria } \\
\text { significativa da pontuação do IPSS total }(-2,12 ; \\
p<0,01) \text { e IPSS subscore miccional }(-1,13 ; p<0,01) \\
\text { Sem diferença estatisticamente significativa no } \\
\text { IPSS subscore de armazenamento }(p=0,06) \\
\text { 2. Sem diferenças estatisticamente significativas } \\
\text { entre os fármacos na pontuação de IPSS total } \\
(-0,11 ; p=0,74) \text {, IPSS subscore miccional } \\
(-0,05 ; p=0,82) \text { e IPSS subscore de armazenamento } \\
(-0,30 ; p=0,45)\end{array}$ & 1 \\
\hline $\begin{array}{l}\text { Brock e } \\
\text { colaboradores } \\
(2014)^{8}\end{array}$ & $\begin{array}{l}4 \text { ECAs } \\
n=1.496 \\
\text { Duplamente } \\
\text { cegos }\end{array}$ & $\begin{array}{l}\text { IPDE5 (tadalafil } 5 \mathrm{mg} / \text { dia) } \\
\text { vs } \\
\text { Placebo }\end{array}$ & $\begin{array}{l}\text { O IPDE5 mostrou-se superior na melhoria do IPSS } \\
\text { total em homens com disfunção eréctil }(-2,3 ; \\
p<0,0001) \text { e nos homens sem disfunção eréctil } \\
(-2,2 ; p=0,0007) \\
\text { Há uma fraca correlação entre a melhoria no IIFE e } \\
\text { a melhoria do IPSS ( } r=-0.29 ; p<0,0001) \text {, o que é } \\
\text { consistente com a hipótese da melhoria do IPSS } \\
\text { ser independente da melhoria do score de } \\
\text { disfunção eréctil. }\end{array}$ & 1 \\
\hline
\end{tabular}

Legenda: $\mathrm{ECA}$ = ensaios clínicos aleatorizados; IPDE5 = inibidores da fosfodiesterase 5; IPSS = International Prostatic System Score; IIFE = The International Index of Erectile Function; $\mathrm{HBP}=$ hipertrofia benigna prostática; $\mathrm{DE}=$ disfunção eréctil; $\mathrm{EA}=$ efeito adverso; $\mathrm{AB}=$ alfa-bloqueantes; $\mathrm{DM}=$ diabetes mellitus; HTA = hipertensão arterial; DCV = doença cardiovascular; Nível de Evidência, segundo a escala SORT da American Family Physician.

3,12 a $-2,07 ; p<0,00001 ;-3,47$ a $-1,68 ; p<0,00001 ;-2,3$ a $-2,90$; $p<0,001)$ e subscore miccional $(-1,13 ; p<0,01 ;-1,4 ; p<0,001)$. Os benefícios no subscore de armazenamento apenas se verificaram no estudo de Porst e colaboradores $(-0,8$; $p<0,001)$. Verificou-se também melhoria no Índice Internacional de Função Eréctil (IIFE) (+5,5; p<0,0001; +4,78 a $+6,70 ; p<0,00001)$ - quadro III.

\section{IPDE5 versus alfa-bloqueante}

Quando comparados os IPDE5 com os AB, não se verificaram diferenças com significado estatístico (IPSS total -0,11; $p=0,74$; IPSS subscore miccional $-0,05 ; p=0,82$ e IPSS subscore de armazenamento $-0,30 ; p=0,45$ ).

\section{$\underline{\text { Perfil de efeitos adversos }}$}

As meta-análises levadas a cabo por Liu e por Porst, bem como pelos colaboradores de ambos, analisaram também os EA do tratamento com IPDE5 tendo ocorrido maior incidência de efeitos adversos leves a moderados, transitórios, nos doentes tratados com estes fármacos - cefaleia, rubor, dispepsia e dor lombar ( $R R+1,87 ; p=0,0005 ; p=0,003)$. A incidência de EA não tem relação com a idade, uso prévio de IPDE5, diabetes mellitus, hipertensão arterial ou doença cardiovascular $(p \geq 0,10)$, mas foi significativamente maior nos que usaram $\mathrm{AB}$ previamente $(p=0,058)$. A incidência de EA graves não foi superior aos grupos controlo $(0,7 \%$ a $0,8 \% ; p=0,766)$.

A todas as meta-análises foi atribuído um Nível de Evidência de 1, pois incluíram estudos aleatorizados, duplamente cegos, com elevado número de participantes, follow-up adequado e outcomes orientados para o doente. 


\begin{tabular}{|c|c|c|c|c|}
\hline Referência & $\begin{array}{l}\text { Estudos, } \\
\text { População }\end{array}$ & Intervenção & Resultados & $\begin{array}{l}\text { Nível de } \\
\text { evidência }\end{array}$ \\
\hline $\begin{array}{l}\text { Liu e } \\
\text { colaboradores } \\
(2011)^{5}\end{array}$ & $\begin{array}{l}11 \text { ECAs } \\
n=2.119 \\
\text { Duplamente } \\
\text { cegos } \\
12 \text { semanas }\end{array}$ & $\begin{array}{l}\text { 1. IPDE5s (sildenafil, } \\
\text { tadalafil, vardenafil) } \\
\text { vs } \\
\text { Placebo } \\
\text { Homens com LUTS/HBP } \\
\text { 2. IPDE5s (sildenafil, } \\
\text { tadalafil, vardenafil) } \\
\text { vs } \\
\text { Placebo } \\
\text { Homens com LUTS/HBP + DE }\end{array}$ & 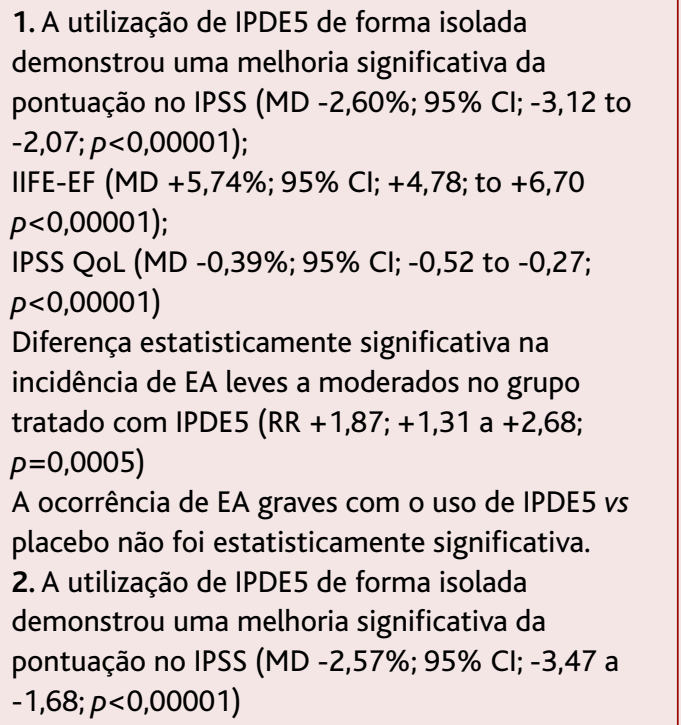 & 1 \\
\hline $\begin{array}{l}\text { Porst e } \\
\text { colaboradores } \\
(2013)^{3}\end{array}$ & $\begin{array}{l}4 \text { ECAs } \\
\mathrm{n}=1.500 \\
\text { Duplamente } \\
\text { cegos } \\
12 \text { semanas }\end{array}$ & $\begin{array}{l}\text { IPDE5 (tadalafil 5mg/dia) } \\
\text { vs } \\
\text { Placebo } \\
\text { Homens com LUTS/HBP, } \\
\text { com ou sem DE }\end{array}$ & $\begin{array}{l}\text { A utilização de tadalafil } 5 \mathrm{mg} / \text { dia demonstrou uma } \\
\text { melhoria significativa da pontuação no IPSS }(-2,3 \text {, } \\
p<0,001) \\
\text { IPSS subscore miccional }(-1,4 ; p<0,001) \\
\text { IPSS subscore de armazenamento }(-0,8 ; p<0,001) \\
\text { IPSS QoL }(-0,4 ; p<0,001) \\
\text { O efeito do tadalafil } 5 \mathrm{mg} / \text { dia foi independente da } \\
\text { idade, uso prévio de AB ou IPDE5, níveis totais de } \\
\text { testosterona ou volume prostático estimado ( } p>0,121) \\
\text { A proporção de EA é comparável entre os subgrupos } \\
\text { independentemente de idade, uso prévio de IPDE5, } \\
\text { DM, HTA ou DCV ( } p \geq 0,10) \text {, mas significativamente } \\
\text { maior nos que usaram AB previamente ( } p=0,058) \\
\text { A proporção de doentes com um ou mais EA leves } \\
\text { a moderados foi significativamente maior no grupo } \\
\text { tratado com tadalafil } 5 \text { mg/dia do que no grupo } \\
\text { placebo ( } p=0,003) \\
\text { A proporção de doentes com um ou mais EA grave } \\
\text { foi semelhante no grupo tratado com tadalafil } \\
5 \text { mg/dia e no grupo placebo ( } 0,7 \% \text { a } 0,8 \% ; p=0,766)\end{array}$ & 1 \\
\hline
\end{tabular}

\section{Revisões sistemáticas}

A RS incluída, realizada por Park e colaboradores em $2013,{ }^{4}$ analisou quatro ECA, duplamente cegos, decorridos num período de 12 semanas, em que um dos braços dos estudos era submetido a terapêutica com tadalafil $5 \mathrm{mg} /$ dia e outro braço a placebo. Serviu para comparar a eficácia e tolerabilidade do tadalafil para os LUTS secundários a HBP, em homens com e sem disfunção eréctil.Verificou-se uma melhoria no IPSS total e nos subscores, tanto em homens com disfunção eréctil (IPSS Total: -6,0; -3,6 $p<0,001$; IPSS subscore miccional: $-3,7 ;-2,1 p<0,01$; IPSS subscore de armazenamento: $-2,3 ;-1,5 p<0,05)$, bem como os que não tinham esta comorbilidade (IPSS total: $-5,4 ;-3,3 p<0,01$; IPSS subscore miccional: $-3,5 ;-2,0 \quad p<0,01$; IPSS subscore arma- 
zenamento: -1,9; -1,3 p<0,05; IPSS QoL: -1,0; -0,7 $p<0,05$ ). A esta RS foi atribuído um Nível de Evidência 1 perante a inclusão de estudos aleatorizados, duplamente cegos, com elevado número de participantes, follow-up adequado e outcomes orientados para o doente.

\section{Ensaios clínicos aleatorizados}

O ECA duplamente cego, desenvolvido por Takeda e colaboradores em 2014, ${ }^{17}$ incluiu 610 homens com 45 anos ou mais, IPSS $\geq 13$, fluxo máximo urinário em urofluxometria (Qmáx) $\geq 4$ (obstrução ao trato de saída vesical), volume vesical pré-miccional entre 150 e $550 \mathrm{~mL}$ e volume prostático $\geq 20 \mathrm{~mL}$. Após um período livre de terapêutica de quatro a oito semanas, todos os doentes foram submetidos a placebo durante quatro semanas, tendo sido randomizados (1:1) a receber tadalafil $5 \mathrm{mg}$ ou placebo por um período de 12 semanas. O objetivo primário foi avaliar a variação do IPSS entre o valor base e depois das 12 semanas de tratamento em cada grupo. Verificou-se melhoria significativa da pontuação no IPSS em relação ao placebo (-6,0 versus $-4,5$; $p<0,001)$. Foi atribuído um Nível de Evidência de 1.

\section{CONCLUSÃO}

A utilização de IPDE5 em monoterapia demonstrou uma melhoria significativa da pontuação no International Prostatic Symptom Score (IPSS) e no International Index of Erectile Function (IIFE). Esta melhoria é independente da idade do doente, IPSS basal, terapêutica prévia, níveis de testosterona ou volume prostático. De notar que a melhoria da função sexual não enviesou a melhoria do IPSS. Na meta-análise que comparou o efeito dos IPDE5 com os $\mathrm{AB}$ verificou-se que foram igualmente eficazes na melhoria do IPSS total e subscores.

Estes achados motivaram a referência destes fármacos nas guidelines da Sociedade Europeia de Urologia para os LUTS moderados a severos em homens com ou sem disfunção eréctil, com uma Força de Recomendação A.

Todos os estudos avaliaram a eficácia das terapêuticas instituídas, mas nem todos analisam os efeitos secundários. Nos estudos que estimaram a incidência destes, o uso de IPDE5 associou-se a uma maior incidência global de EA de grau leve a moderado - rubor, cefaleias, dispepsia e dorsalgias, mas sem que tivesse sido observado um aumento significativo da incidência de EA graves. Assim, o perfil de segurança desta terapêutica foi favorável.

Apesar da evidência favorável para a utilização dos IPDE5 como terapêutica dos LUTS na HBP, os estudos que a suportam foram realizados por períodos de tempo inferiores a um ano, na sua grande maioria por períodos de cer- ca de 12 semanas. Deste modo, não é possível inferir resultados a longo prazo, quer na progressão da doença quer no perfil de segurança da utilização crónica destes fármacos. Também não foram, ainda, desenvolvidos estudos de custo-efetividade, que são de importância vital, tendo em vista o custo elevado de comercialização dos mesmos.

Perante estas limitações, mas tendo em conta a qualidade e o rigor com que foram realizados os estudos apresentados, há evidência suficiente para afirmar que os IPDE5 são eficazes e seguros no tratamento dos LUTS secundários a HBP - Força de Recomendação A.

Existe, ainda, a necessidade de realização num futuro próximo de mais estudos aleatorizados, duplamente cegos, prospetivos com amostras relevantes, para perceber o impacto e a segurança da terapêutica com IPDE5 a longo prazo.

\section{REFERÊNCIAS BIBLIOGRÁFICAS}

1. Füllhase C, Chapple C, Cornu JN, De Nunzio C, Gratzke C, Kaplan SA, et al. Systematic review of combination drug therapy for non-neurogenic male lower urinary tract symptoms. Eur Urol. 2013;64(2):228-43.

2. Direção-Geral da Saúde. Abordagem terapêutica farmacológica da hipertrofia benigna da próstata: norma $n^{\circ}$ 048/2011, de 26/12/2011. Lisboa: DGS; 2011.

3. Porst H, Oelke M, Goldfischer ER, Cox D, Watts S, Dey D, et al. Efficacy and safety of tadalafil $5 \mathrm{mg}$ once daily for lower urinary tract symptoms suggestive of benign prostatic hyperplasia: subgroup analysis of pooled data from 4 multinational, randomized, placebo-controlled clinical studies. Urology. 2013;82(3):667-73

4. Park H,Won JE, Sorsaburu S, Rivera PD, Lee SW. Urinary tract symptoms (LUTS) secondary to benign prostatic hyperplasia (BPH) and LUTS/BPH with erectile dysfunction in Asian men: a systematic review focusing on tadalafil. World J Mens Health. 2013;31(3):193-207.

5. Liu L, Zheng S, Han P, Wei Q. Phosphodiesterase-5 inhibitorsfor lower urinary tract symptoms secondary to benign prostatic hyperplasia: a systematic review and meta-analysis. Urology. 2011;77(1):123-9.

6. Gacci M, Corona G, Salvi M, Vignozzi L, McVary KT, Kaplan SA, et al. A systematic review and meta-analysis on the use of phosphodiesterase 5 inhibitors alone or in combination with a-blockers for lower urinary tract symptoms due to benign prostatic hyperplasia. Eur Urol. 2012;61:994-1003.

7. Brock GB, McVary KT, Roehrborn CG, Watts S, Ni X, Viktrup L, et al. Direct effects of tadalafil on lower urinary tract symptoms versus indirect effects mediated through erectile dysfunction symptom improvement: integrated data analyses from 4 placebo controlled clinical studies. J Urol. 2014;191(2):405-11.

8. Yan $\mathrm{H}$, Zong $\mathrm{H}, \mathrm{Cui}$ Y, Li N, Zhang Y. The efficacy of PDE5 inhibitors alone or in combination with alpha-blockers for the treatment of erectile dysfunction and lower urinary tract symptoms due to benign prostatic hyperplasia: a systematic review and meta-analysis. J Sex Med. 2014;11(6):1539-45.

9. Kramer A. Disfunção erétil [cited 2016 Apr 20]. In: BMJ Best Practice [Internet]. 2014 Sep 24. Available from: http://portugal.bestpractice.bmj.com/best-practice/monograph/213.html

10. Sairam K, Kulinskaya E, McNicholas TA, Boustead GB, Hanbury DC. Sildenafil influences lower urinary tract symptoms. Br J Urol. 2002;90(9):836-9.

11. Mulhall JP, Guhring P, Parker M, Hopps C.Assessment of the impact of sildenafil citrate on lower urinary tract symptoms in men with erectile dysfunction. J Sex Med. 2006;3(4):662-7.

12. McVary KT, Roehrborn CG, Kaminetsky JC, Auerbach SM, Wachs B, Young JM, et al. Tadalafil relieves lower urinary tract symptoms secondary to benign prostatic 
hyperplasia. J Urol. 2007;177(4):1401-7.

13. Laydner HK, Oliveira P, Oliveira CR, Makarawo TP, Andrade WS, Tannus M, et al. Phosphodiesterase 5 inhibitors for lower urinary tract symptoms secondary to benign prostatic hyperplasia: a systematic review. BJU Int. 2011;107(7):1104-9.

14. Ebell MH, Siwek J, Weiss BD, Woolf SH, Susman J, Ewigman B, et al. Strength of Recommendation Taxonomy (SORT): a patient-centered approach to grading evidence in the medical literature. Am Fam Physician. 2004;69(3):548-56.

15. Gravas S, Bach T, Bachmann A, Drake M, Gacci M, Gratzke C, et al. Guidelines on the management of non-neurogenic male lower urinary tract symptoms (LUTS), incl. benign prostatic obstruction (BPO) [Internet]. Arnhem, NL: European Association of Urology; 2015. Available from: https://uroweb.org/wp-content/uploads/EAU-Guidelines-Non-Neurogenic-Male-LUTS-Guidelines-2015v2.pdf

16. Wang X, Wang X, Li S, Meng Z, Liu T, Zhang X. Comparative effectiveness of oral drug therapies for lower urinary tract symptoms due to benign prostatic hyperplasia: a systematic review and network meta-analysis. PLOS ONE. 2014;9(9):e107593.

17. Takeda M, Yokoyama O, Lee SW, Murakami M, Morisaki Y, Viktrup L. Tadalafil 5 mg once-daily therapy for men with lower urinary tract symptoms suggestive of benign prostatic hyperplasia: results from a randomized, double-blind, placebo-controlled trial carried out in Japan and Korea. Int J Urol. 2014;21(7):670-5.

\section{CONFLITO DE INTERESSES}

Os autores declaram não ter conflitos de interesses.

\section{ENDEREÇO PARA CORRESPONDÊNCIA}

Hugo Rocha

R. Senhora do Porto, $343-2^{\circ} \mathrm{C}, 4250-455$ Porto

E-mail: hugofp.rocha@gmail.com

Recebido em 23-10-2015

Aceite para publicação em 03-06-2016

\section{ABSTRACT}

\section{THE ROLE OF PHOSPHODIESTERASE 5 INHIBITORS IN THE TREATMENT OF LOWER URINARY TRACT SYMPTOMS: AN EVIDENCE BASED REVIEW}

Aim: The aim of this study was to review the evidence for the efficacy and safety of monotherapy with phosphodiesterase 5 inhibitors (PDE5I) in the treatment of lower urinary tract symptoms (LUTS) due to prostatic hyperplasia in men with or without erectile dysfunction.

Data Sources: MEDLINE and evidence-based medicine sites.

Review methods: We searched clinical guidelines, meta-analyses, systematic reviews and randomized clinical trials using the MeSH terms: 'Phosphodiesterase 5 inhibitors', 'Lower urinary tract symptoms', 'Prostatic hyperplasia'. To assess the quality and strength of recommendation of the trials we used the Strength of Recommendation Taxonomy (SORT) scale, from the American Family Physician. Results: We found 172 articles and selected eight for this review. One clinical guideline, five meta-analyses, one systematic review, and one randomized clinical trial met the inclusion criteria. The use of PDE5I resulted in significant improvement in the International Prostatic Symptom Score (IPSS) and International Index of Erectile Function (IIFE) score. They were associated with a higher overall incidence of mild to moderate adverse events, with no differences in serious adverse events, providing a favorable safety profile.

Conclusions: There is strong evidence for the efficacy and safety of the PDE5I in the treatment of LUTS, with or without associated erectile dysfunction (SORT A). We suggest conducting further studies to evaluate the long-term efficacy and safety of this treatment, their effect on the progression of disease and their cost-effectiveness in the treatment of LUTS.

Keywords: Lower urinary tract symptoms; LUTS; Phosphodiesterase 5 inhibitors; Prostatic hyperplasia. 